\title{
MALDI-TOF-MS Analysis of Bacterial Spores: Wet Heat-Treatment as a New Releasing Technique for Biomarkers and the Influence of Different Experimental Parameters and Microbiological Handling
}

\author{
Verena Horneffer, ${ }^{*}$ Johan Haverkamp and Hans-Gerd Janssen \\ Advanced Measurement and Imaging, Unilever R \& D, Vlaardingen, The Netherlands \\ Regina Notz \\ Microbiological Control Foods Research Center, Unilever R \& D, Vlaardingen, The Netherlands
}

\begin{abstract}
Short wet heat-treatment is presented as a new technique to release high-mass biomarkers to obtain strain-specific fingerprints of intact bacterial spores by matrix-assisted laser desorption/ionization-mass spectrometry (MALDI-MS). Wet heat-treatment was applied for several minutes (3-30) by two techniques using either a screw-cap tube submerged in a glycerol bath at $120^{\circ} \mathrm{C}$ or an Eppendorff-tube submerged in a water bath at $100{ }^{\circ} \mathrm{C}$. Both techniques turned out to be successful for releasing high-mass biomarkers. The influence of different experimental parameters and microbiological handling on the peak pattern of the released high-mass biomarkers was studied. While the sporulation medium, the applied washing procedure, and the choice of matrix crucially influenced the peak pattern, other parameters like storage conditions were found to be insignificant. A protocol of optimized experimental conditions for MALDI-MS of wet heat-treated spores is presented. (J Am Soc Mass Spectrom 2004, 15, 1444-1454) (C) 2004 American Society for Mass Spectrometry
\end{abstract}

$\mathrm{F}$ or food, environmental, and medical hazard control, rapid identification and characterization of bacterial cells and spores is of great relevance and finds large interest. Analyses of physiological and biochemical traits, immunodiagnostic assays, and nucleic acid analysis have been used to identify bacteria in the past. Next to this, mass spectrometry (MS) has been applied in several ways for the analysis of bacterial cells. The first paper dates back to 1975 when gently heat-treated bacteria were analyzed by MS [1]. Small molecules served as biomarkers [2]. With the modern soft ionization methods, e.g., Matrix-assisted laser desorption/ionization (MALDI) and electrospray-ionization (ESI), fragile biopolymeric marker molecules with masses higher than $4000 \mathrm{u}$ (high-mass biomarkers) have become accessible for MS analysis. Lysates of bacterial cells were analyzed with MALDI-MS in 1994 [3]. Several reports about rapid identification of intact whole

Published online September 11, 2004

Address reprint requests to Dr. V. M. Horneffer, Medical Laser Center Lübeck, Peter-Monnik-Weg 4, D-23562 Lübeck, Germany. E-mail: horneffer@mll.mu-luebeck.de

* Current address: Same as above. bacteria with MALDI-MS were published in the past years [4-7]. In a recent study, Williams et al. investigated the influence of experimental factors on the analysis of whole bacteria with MALDI-MS and demonstrated that various experimental parameters significantly affect the quality and reproducibility of the mass spectra [8]. In spite of this, the implementation of MS for the analysis of bacterial cells and spores has grown in such a remarkable speed that commercial databases have been developed recently for rapid screening and identification, e.g., MicrobeLynx by Micromass/Waters.

The application of MS to bacterial spores is much more problematic because of the complex structure of the spores [9]. It was observed that the release of useful biomarkers is a general problem in the analysis of bacterial spores [10]. Different techniques have been evaluated to improve the detection of high-mass biomarkers [11, 12]. Birmingham et al. released high-mass biomarkers from bacterial spores by pretreating the spores with corona plasma discharge or sonication [11]. Subsequently, strong acid treatment (acidic extraction of high-mass biomarkers) was suggested by Elhanany et al. [12]. Using this techniques, bacterial spores could 
be discriminated by means of their MALDI biomarker pattern at the strain and species level [13-15]. The latest application uses bioactive glass slides to simplify the analysis of bacterial spores by specific surface absorption and lysing the spores with strong acids [16]. Most techniques so far applied damage the spore wall severely [11-16]. This enables the release of components from the coat or other interior compartments on the one hand but can cause difficulties in the subsequent analytical procedure on the other hand. Therefore, a more convenient, less detrimental releasing technique to pretreat bacterial spores for mass spectrometric analysis is desired.

In this study, wet heat-treatment will be shown as a new, soft releasing technique for high-mass biomarkers from intact bacterial spores which can be directly analyzed by MALDI-MS. The masses of the released biomarkers acquired with a time-of-flight (TOF) MALDI-MS instrument are compared with masses obtained by other releasing techniques. The influence of different experimental parameters on the biomarker peak pattern is discussed in respect of literature. Preliminary results of the influence of microbiological handling on the biomarker patterns are presented.

\section{Experimental}

\section{Materials}

The following product isolates were used in this study: Bacillus subtilis (strains MC85 and RL45), Bacillus cereus (strain PEA26), and Bacillus sporothermodurans (strain IC4). The strains were identified using the API50CHB and API20E systems with the help of APILAB Plus software version 3.3.3 (BioMérieux, Mercy, France). In addition, the results were confirmed by sequencing their 16S RNA. Bacteria were routinely stored in 50\% glycerol (wt/wt) at $-80{ }^{\circ} \mathrm{C}$ in the culture collection of Unilever Research Vlaardingen. The following substances were tested as MALDI matrices: Three different lots of 3,5-dimethoxy-4-hydroxycinnamic acid (sinapinic acid, SA; two from Fluka, Buchs, Switzerland, analysis numbers 376708/1 61498 and 47353/2 31698 and one from Aldrich, Milwaukee, WI), $\alpha$-cyano-4hydroxycinnamic acid (HCCA, Aldrich), 2,4,6-trihydroxyacetophenon (2,4,6-THAP, Fluka), 2,5-dihydroxybenozoic acid (2,5-DHB) and 2-hydroxy-5methoxybenzoic acid, both necessary for the matrix mixture DHBs, (Sigma, Steinheim, Germany). All matrix substances were of technical grade (i.e., $>90 \%$ purity) and were used without further purification. Calibrating substances were renin substrate, insulin from bovine pancreas, bovine ubiquitin, and horse heart cytochrome $c$, all supplied by Sigma. Acetonitrile was purchased from Biosolv LtD (Valkenswaard, The Netherlands), and ethanol from Merck (Darmstadt, Germany) both in HPLC-grade. Trifluoroacetic acid was supplied by J.T. Baker (Deventer, The Netherlands).

\section{Methods}

Sporulation conditions. Sporulation was obtained on nutrient agar (Becton, Dickinson and Co., Cockeyville, MD) supplemented with a metal mix containing $\mathrm{MgSO}_{4}, \mathrm{KCl}, \mathrm{CaCl}_{2}, \mathrm{MnSO}_{4}$, and $\mathrm{FeSO}_{4}$ as described by Cazemier et al. [17]. $100 \mu \mathrm{l}$ of bacterial stock was spread on nutrient agar plates, which were then incubated at $37{ }^{\circ} \mathrm{C}$ for five days. At this point, more than $90 \%$ of the material consisted of phase-bright spores. These were harvested and washed three times with sterile deionized water at ambient temperature. The remaining vegetative cells were killed by pasteurization of the samples for $10 \mathrm{~min}$ at $80{ }^{\circ} \mathrm{C}$. The concentration of spores was microscopically quantified with a Burker-Turk counting chamber. The quantity of colony forming units per $\mathrm{ml}$ was estimated by plating serial dilutions on trypticase soy agar (TSA, Becton, Dickinson and Co.). The spore solutions were then stored in aqueous solutions either at 4 or at $-80{ }^{\circ} \mathrm{C}$ until further use. Frozen spores were carefully thawed at $4{ }^{\circ} \mathrm{C}$ for several hours. Schaeffer's sporulation medium [18] and brain heart infusion agar (BHI, Becton, Dickinson and Co.) were in some cases used to obtain spore crops.

Wet Heat-treatment techniques. Wet heat-treatment of aliquots of spore solutions was carried out following two different techniques: (1) The screw-cap tube method described by Kooiman [19] where $200 \mu \mathrm{l}$ of a spore suspension were injected into $9.8 \mathrm{ml}$ deionized water preheated in $10 \mathrm{ml}$ stainless steel screw-cap tubes in a glycerol-bath at $120{ }^{\circ} \mathrm{C}$. After heating for $3 \mathrm{~min}$ and $20 \mathrm{~min}$, respectively, samples were cooled immediately in a mix of ice and water. This technique will be referred to as the Kooiman method throughout this study. (2) A simpler wet heat-treatment was performed by heating $50 \mu \mathrm{l}$ aliquots of spores solutions in conventional Eppendorff-reaction tubes in a water bath at $100{ }^{\circ} \mathrm{C}$ for 15 to $30 \mathrm{~min}$.

Further actions on the wet heat-treated spore solutions. For the Kooiman method, the quantity of heat-injured spores was estimated by plating serial dilutions of treated and untreated aliquots on TSA. Wet heat-treated spores were microscopically requantified with a Burker-Turk counting chamber and stored in deionized water at either $4{ }^{\circ} \mathrm{C}$ or $-80^{\circ} \mathrm{C}$ and protected from light until further analysis. All spore samples were diluted with deionized water to a final concentration of approximately $10^{9}$ spores $/ \mathrm{ml}$ for MALDI analysis. For experiments dealing with the influence of concentration, samples were diluted 1-3 times by a factor of 10 with deionized water. Spores were washed up to three times with deionized water.

Acidic extraction. High-mass biomarker extraction of selected spores was performed following the protocol of Elhanany et al. [12]. 
Matrix solutions. $10 \mathrm{mg}$ of SA and HCCA were dissolved in $1 \mathrm{ml}$ acetonitrile $/ 0.1 \%$ trifluoroacetic acid in water $(70: 30, \mathrm{vol} / \mathrm{vol})$. A solution of 2,4,6-THAP was produced in acetonitrile/deionized water (50:50, vol/ vol) at a concentration of $10 \mathrm{mg} / \mathrm{ml}$. For DHBs-matrix mixture, nine volumes of a $20 \mathrm{mg} / \mathrm{ml}$ concentrated 2,5-DHB solution in deionized water were mixed with one volume of a $20 \mathrm{mg} / \mathrm{ml}$ concentrated 2-hydroxy-5methoxybenzoic acid solution in pure ethanol.

Sample preparation. Two standard preparation techniques were tested, a) the inverse two-layer standard preparation technique (inverse two-layer method) [13, 20] and b) the so-called dried-droplet method [21]. Every sample was prepared at least in duplicate.

\section{Instrumentation}

All MALDI spectra were recorded on a TofSpec-2E TOF mass spectrometer (Micromass, Manchester, UK) extracting positive ions in linear mode with delayed extraction. $20 \mathrm{kV}$ was applied as acceleration voltage. Ionization was initiated with a nitrogen laser operating at $337 \mathrm{~nm}$ with a repetition rate of $3 \mathrm{~Hz}$. The laser fluence was checked for selected samples and an average value was chosen for the automatic acquisition. A mass range up to $m / z 14,000$ was scanned while ions up to $\mathrm{m} / \mathrm{z} 1000$ were suppressed. Twenty spectra from different spots were summed for each sample and each spectrum comprised the ions of 50 laser shots. All spectra were manually processed, e.g., background subtraction and smoothing, using the MassLynx 3.4 software package. The external calibration was based on renin substrate, bovine insulin, and horse heart cytochrome $c$. Ubiquitin was used as an internal standard. The intensities in all shown spectra are normalized with respect to the most intense peak of the shown $m / z$ section.

\section{Results and Discussion}

\section{Wet Heat-Treatment as a New Releasing Technique}

As seen in Figures 1, 2, 3, and 4, wet heat-treatment indeed enables the release of strain-specific high-mass biomarkers from intact spores and allows their detection with MALDI-TOF-MS. Figures 1, 2, and 3 show the biomarker pattern of three different Bacillus species: $B$. subtilis strain MC85 (Figure 1), B. cereus strain PEA26 (Figure 2), and B. sporothermodurans strain IC4 (Figure 3). In each of the figures, a mass spectrum of the untreated spore solution after pasteurization (c in the Figures) is compared with the mass spectra of spores, which were wet heat-treated with the Kooiman method $\left(120{ }^{\circ} \mathrm{C}\right.$ ) for $3 \mathrm{~min}(\mathrm{~b}$ in the Figures) and $20 \mathrm{~min}$ ( $\mathrm{a}$ in the Figures). Sinapinic acid and the inverse two-layer method were used for the preparation, which turned out as best experimental parameters (see below). The

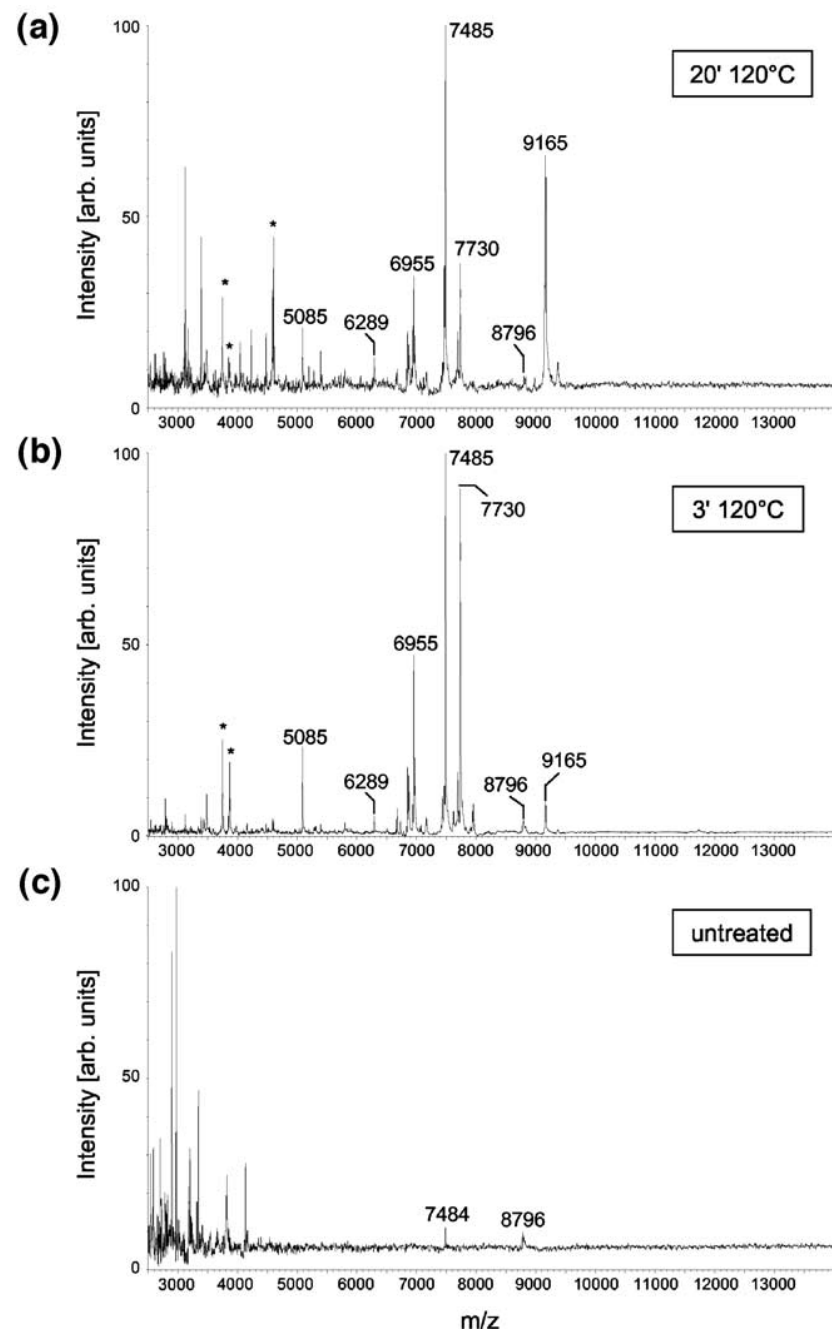

Figure 1. MALDI mass spectrum of pasteurized B. subtilis strain MC85 after different duration of wet heat-treatment at $120^{\circ} \mathrm{C}$. (a) $20 \mathrm{~min}$, (b) $3 \mathrm{~min}$, and (c) $0 \mathrm{~min}$. SA was used as matrix and the inverse two-layer method as preparation technique. The asterisk indicates peak of doubly charged species.

high-mass biomarkers pattern obtained after wet heattreatment is highly reproducible; at least $95 \%$ within 10 independent experiments for both, wet heat-treatment and MALDI-MS measurement.

In general, the spectra of untreated (i.e., only pasteurized) spore solutions show a large number of random peaks in a mass range up to $m / z 4000$, while strain specific peaks around $\mathrm{m} / \mathrm{z} 4000$ to 10,000 only appear after wet heat-treatment with the Kooiman method. The signal-to-noise ratio $(\mathrm{S} / \mathrm{N})$ ratio of these high-mass biomarkers increased at least by a factor of 10 when applying the Kooiman method. This observation also holds for the other Bacillus species and strains tested (data not shown). It was observed that spores of all strains still had the ability to germinate after a $3 \mathrm{~min}$ wet heat-treatment if they were put on a suitable recovery medium. Experiments with strain IC4 showed that after wet heat-treatment even more spores were able to grow out than without wet heat-treatment (data 
(a)

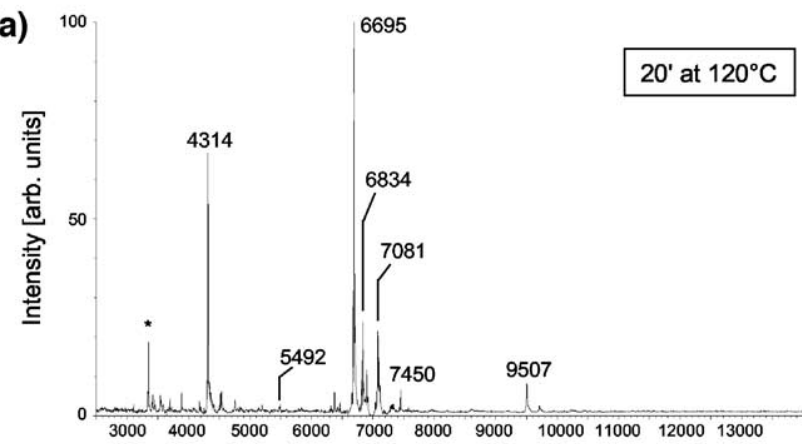

(b)

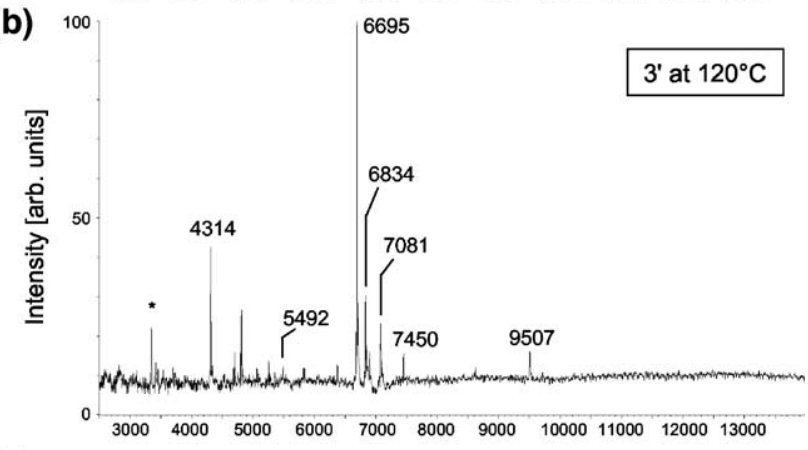

(c)

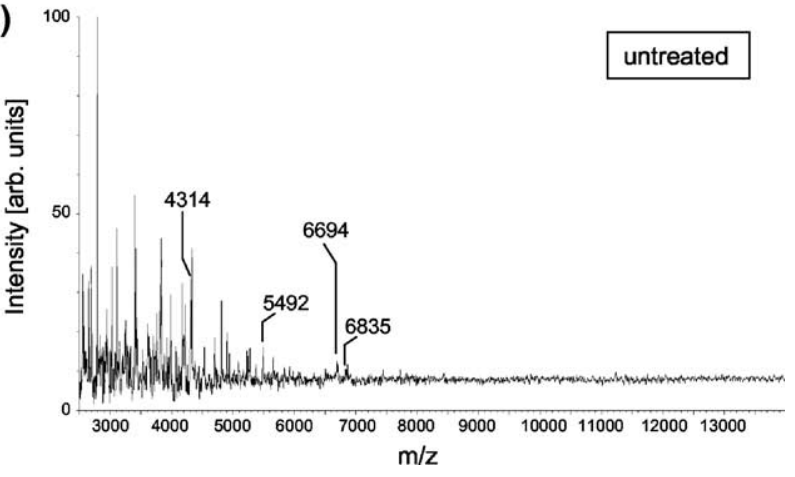

Figure 2. MALDI mass spectrum of pasteurized B. cereus strain PEA26 after different duration of wet heat-treatment at $120^{\circ} \mathrm{C}$. (a) $20 \mathrm{~min}$, (b) $3 \mathrm{~min}$, and (c) $0 \mathrm{~min}$. SA was used as matrix and the inverse two-layer method as preparation technique. The asterisk indicates peak of doubly charged species.

not shown). Longer wet heat-treatment led to a higher die-off of the spores. Interestingly, the spectrum quality, i.e., the appearance of the high-mass biomarkers, increased with the length of duration of wet heattreatment for most of the samples (e.g., Figure 2 and Figure 3). This observation might be of advantage for further microbiological investigations, e.g., for correlating the biomarker peak pattern to the degree of injury and die-off of the spores. A more thorough study into the feasibility of injury typing of wet heat-treated spores with MALDI-TOF-MS will be presented in a separate publication [22].

Initial experiments were performed at $120{ }^{\circ} \mathrm{C}$ because the Kooiman method routinely used in-house to simulate preservation by wet-heat uses this temperature. It was investigated then whether temperatures lower than $120^{\circ} \mathrm{C}$ are suitable to release the strainspecific high-mass biomarkers. This was performed by (a)

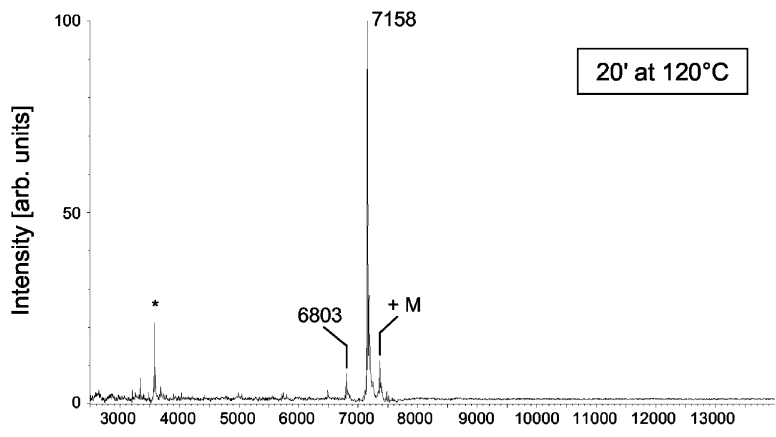

(b)

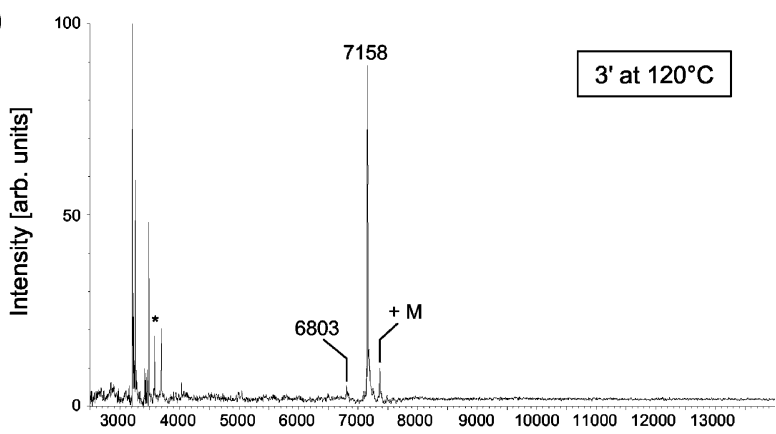

(c)

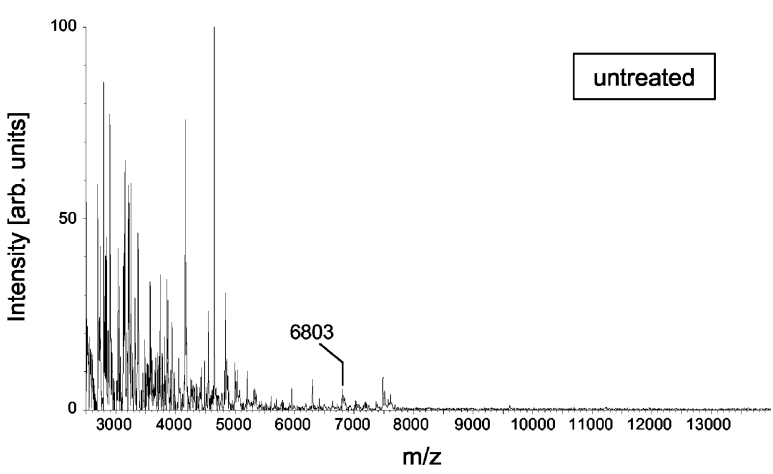

Figure 3. MALDI mass spectrum of pasteurized B. sporothermodurans strain IC4 after different duration of wet heat-treatment at $120{ }^{\circ} \mathrm{C}$. (a) $20 \mathrm{~min}$, (b) $3 \mathrm{~min}$, and (c) $0 \mathrm{~min}$. SA was used as matrix and the inverse two-layer method as preparation technique. The asterisk indicates peak of doubly charged species, $+\mathrm{M}$ indicates peak due to matrix adduction.

boiling aliquots of the spore solution in a water bath for several minutes. As an example, Figure 4 shows the mass spectra of the B. cereus strain PEA26 spores after 15 min wet heat-treatment at $100{ }^{\circ} \mathrm{C}$ in a boiling water bath. Again, the peak pattern of the wet heat-treated spores strongly differs from the untreated spores (Figure 2c) and contains the characteristic signals of the high-mass biomarkers. The $\mathrm{m} / \mathrm{z}$ values observed are identical to those obtained with the Kooiman method, Figure $2 a$ and $b$. This observation was true for all other strains tested (data not shown). As this technique was applied only as an alternative to the Kooiman method, no detailed investigation on the viability of the spores was performed.

Average molecular masses of selected high-mass biomarkers of each strain released by wet heat-treatment techniques are given in Table 1. A standard deviation of 200 ppm was attained for all masses by 


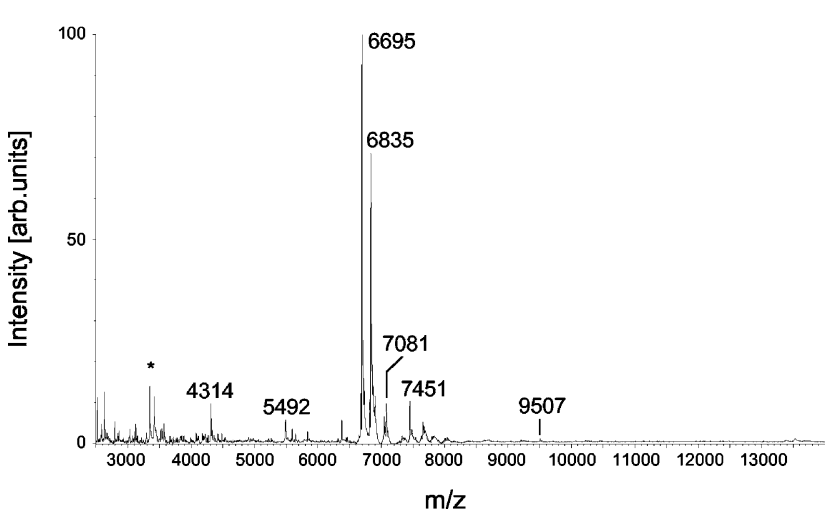

Figure 4. MALDI mass spectrum of B. cereus strain PEA26 after wet heat-treated at $100{ }^{\circ} \mathrm{C}$ for $15 \mathrm{~min}$. SA was used as matrix and the inverse two-layer method as preparation technique. The asterisk indicates peak of doubly charged species.

internal calibration with bovine ubiquitin. A good intralaboratory congruence was found also for different strains (MC85 and RL45) of B. subtilis showing peaks at $\mathrm{m} / \mathrm{z}$ 6849, 6955, 7485, 7730, and 9166.

So far, the detection of high-mass biomarkers directly from intact bacterial spores was only possible by treating the spores with sonication or corona plasma discharge (CPD). These techniques were introduced and successfully applied by the group of Fenselau [11, 14]. These techniques have not been applied in our work. Elhanany suggested to release high-mass biomarkers from the spores by acidic extraction in a solution of ACN/0.1\%TFA (30/70 vol/vol) for $10 \mathrm{~min}$ at room temperature [12]. Although this was repeatedly applied to our bacterial spores, we were not able to obtain mass spectra containing signals of high-mass biomarkers with this technique (data not shown). Compared with our wet heat-treatment techniques, the conditions applied with CPD, sonication, and acidic extraction are quite harsh, leading to changes or mechanical destruction of the spore wall $[11,12,15,23]$. On the contrary, wet heat-treatment did not lead to changes of the spore membrane as evidenced by microscopic inspection during counting. In a recent study, Leuschner et al. showed that the outer spore wall remained intact after wet heat-treatment for several hours at $60{ }^{\circ} \mathrm{C}$ to $80{ }^{\circ} \mathrm{C}$ [24]. Therefore, wet heat-treatment appears to be much milder and allows further investigations of the intact spores.

Selected masses of similar strains measured with the CPD-technique or after acidic extraction are listed in Table 1 for comparison [12, 14]. $\mathrm{m} / \mathrm{z}$ Values found within a deviation of $500 \mathrm{ppm}$ for different strains are given in bold. For instance, the $\mathrm{m} / \mathrm{z}$ values of high-mass biomarkers of the B. cereus strain PEA26 at $\mathrm{m} / \mathrm{z}$ 6695, 6834, 7450, and 7081 obtained with both of our wet heat-treatment techniques (Figures 2 and 4 ) are identical to those of B. cereus strain B33 obtained with CPD [14]. Some of the other peaks were also identified by CPD or acidic extraction $[12,14]$. Unfortunately, no data of $B$. sporothermodurans were available in literature. However, notable differences in peak patterns are observed for identical species but different strains. This cannot be explained just by general measurement inaccuracy. It is speculated that these discrepancies are resulting from strain-specific properties of the highmass biomarkers within a bacterial species. It is, therefore, assumed that the high-mass biomarker peak pattern released by wet heat-treatment can serve well for obtaining specific fingerprints of individual bacterial strains.

Recently, the high-mass biomarkers found by MALDI analysis after CPD treatment could be linked to masses of so-called small acid-soluble protein (SASPs) published in protein databases [13-15]. Based on the strong similarity of $\mathrm{m} / \mathrm{z}$ pattern we speculate that the high-mass biomarkers released by our wet heat-treatment techniques also represent SASPs. It is, however, very surprising that short wet heat-treatment releases SASPs from the spore core, which is surrounded by several strong layers, e.g., the coat and further membranes $[9,25]$. Further investigation to understand the

Table 1. Observed average masses of high-mass biomarkers for Bacillus strains with SA as matrix and inverse two-layer method. All masses have a standard deviation of 200 ppm (at least 10 measurements). Biomarker peaks with a similar mass (deviation with 500 ppm) observed for different strains are given in bold.

\begin{tabular}{|c|c|c|c|c|c|c|c|}
\hline \multicolumn{4}{|c|}{ B. subtilis } & \multicolumn{3}{|c|}{ B. cereus } & \multirow{2}{*}{$\begin{array}{c}\text { B. sporothermodurans } \\
\text { IC4 }\end{array}$} \\
\hline MC85 & RL45 & EMG 168 [14] & 168 [12] & PEA26 & B33 [14] & $\mathrm{T}[12]$ & \\
\hline 5086 & 5085 & & 4585 & 3348 & & & \\
\hline 5196 & 5140 & & & 4314 & 4333 & & \\
\hline 5391 & 5961 & & 5802 & & 4833 & & 4952 \\
\hline 6290 & 6289 & & & 5492 & & 6362 & 5441 \\
\hline 6673 & 6673 & & & 6695 & 6695 & 6698 & \\
\hline 6850 & 6849 & 6847 & & 6835 & 6834 & 6820 & 6803 \\
\hline 6944 & 6955 & 6949 & 6939 & & & & \\
\hline 7485 & 7485 & & & 7081 & 7082 & 7065 & 7158 \\
\hline 7731 & 7730 & & & 7452 & 7451 & & \\
\hline & 7955 & & & & & & \\
\hline 8796 & 8767 & & & & & & \\
\hline 9166 & 9165 & 9136 & & 9507 & & 9519 & \\
\hline
\end{tabular}


release of these biomarkers from intact spore by wet heat-treatment is, therefore, necessary.

Nevertheless, our wet heat-treatment techniques are suitable and more gentle alternatives to release highmass biomarker molecules for the analysis with MALDI-MS from intact bacterial spores. Wet heattreatment, especially in a water bath, is a simple procedure to provide strain-specific fingerprints which can be carried out reproducibly and without specialized equipment in any laboratory. Using this method in further investigations might provide new opportunities to understand the spore biophysics by correlating the peak pattern to other spores- or strain-specific data, such as the viability of the spores after wet heattreatment.

\section{Influences of Mass Spectrometric Parameters}

Several different parameters of the mass spectrometric handling were tested for their possible influence on the peak pattern in MALDI spectra using wet heat-treatment as high-mass biomarker releasing technique.

\section{MALDI-Preparation Techniques}

It is well known that the quality of a MALDI spectrum crucially depends on the combination of the matrix chosen and the sample preparation technique applied. Two preparation techniques, the inverse two-layer method and the dried-droplet method, frequently used in literature were tested in combination with SA and DHBs as matrix substances [11, 13-15, 26-33]. Our results were compared with respect to spectrum quality, e.g., S/N ratio, matrix background, and spectral reproducibility (spot-to-spot and preparation-to-preparation).

Inverse-two layer. Here, the morphology of the preparation is strongly determined by the first layer of spore solution. Most of the spore suspensions left a circle with a thin homogeneous inner part and a thick outer rim. Only spores of strain PEA26 formed clusters on the target, which can be explained by the more hydrophobic surface of $B$. cereus spores compared with other Bacillus species [34, 35]. After addition of the matrix solution, the preparation morphology became very homogeneous if SA was used as matrix substance. Good quality mass spectra were achieved with excellent preparation-to-preparation reproducibility and with good spot-to-spot reproducibility for the microcrystalline inner part (data not shown). In contrast, DHBs formed large crystals on top of the preparation rim and a microcrystalline inner part. It should be noted that the quality of the crystals at the preparation rim is worse than for conventional DHBs preparations when used for the analysis of standards (neat protein solutions). Good quality spectra were obtained from certain crystals of the rim, whereas the inner part delivered spectra with unacceptable high background signals and a poor $\mathrm{S} / \mathrm{N}$ ratio.

Dried-droplet. The resulting morphology was more homogeneous for both matrices over the entire area due to the missing rim of dried spores, but more macrocrystalline, especially with bigger crystals at the rim of the preparation. For SA, this preparation technique provided comparable results in terms of spectra quality and preparation-to-preparation reproducibility and was worse with respect to spot-to-spot reproducibility than the inverse two-layer method. For DHBs, this preparation technique was unsuccessful because the crystallization process was disturbed and larger crystals could not be established; no mass spectra of good quality could be obtained. Consequently, it was decided to use the inverse two-layer method as standard experimental conditions for studies towards MALDI-MS analysis of wet heat-treated spores.

\section{Choice of MALDI-Matrix}

SA was tested as matrix substance first because it is commonly used for the analysis of high-mass biomarkers in literature [12-15, 36]. In addition, other standard matrices were tested, which are known for their differing properties as matrix substance. HCCA was chosen as an alternative matrix for SA [37], 2,4,6-THAP because of its suitability for the analysis of fragile molecules such as oligonucleotides and non-covalent complexes $[38,39]$. DHBs was the last matrix tested. This matrix mixture is known for its versatility and its properties are studied best next to those of SA [21, 30, 32, 40-45]. As an example, Figure 5 shows mass spectra of a $B$. subtilis strain RL45 after wet heat-treatment with the Kooiman method $\left(20 \mathrm{~min}\right.$ at $120^{\circ} \mathrm{C}$ ) using different matrix substances. The inverse two-layer method was used for all matrices. It is notable that there is a strong influence of the matrix on the peak pattern of the high-mass biomarkers for the identical sample solution. Although some differences could be expected, this strong matrix effect is remarkable. The mass spectrum with SA as matrix (Figure 5a) is similar to that of $B$. subtilis strain MC85 in Figure 1a), which can be explained by the close relation of the two strains RL45 and MC85. The mass spectrum with HCCA as matrix (Figure $5 b$ ) shows much more peaks in the lower $m / z$ range, e.g., $m / z$ 3387, 5031, 5195, 5297, and 5803, while some major peaks in the higher $\mathrm{m} / \mathrm{z}$ range are missing, e.g., $m / z 7730$ and 7955 . The intensity pattern of the major peaks $(m / z 6955,7485$, and 9166) is comparable with that obtained with SA as matrix. 2,4,6-THAP shows a mass spectrum (Figure 5c) dominated by peaks at $m / z$ 5961, 7730, and 9166 together with their doubly charged species. DHBs provides a peak pattern highly comparable to that obtained with SA but with additional peaks in the $m / z$ range 9000 to 10,000 (Figure $5 d$ ). These peaks can not be explained by matrix adducts because (1) the differences in $m / z$ values (198 and 355) are not 
(a)

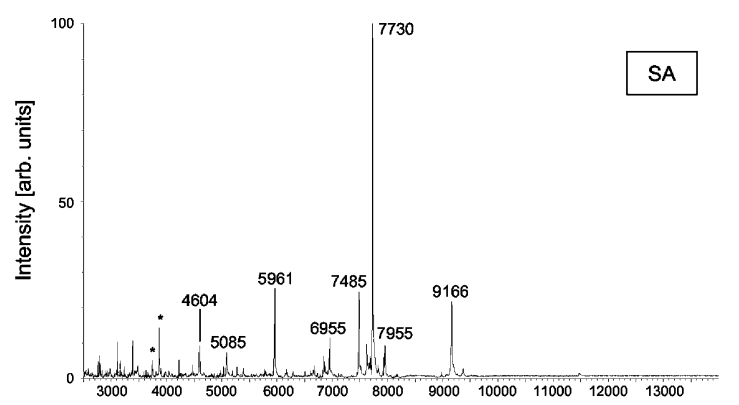

(b)

(c)

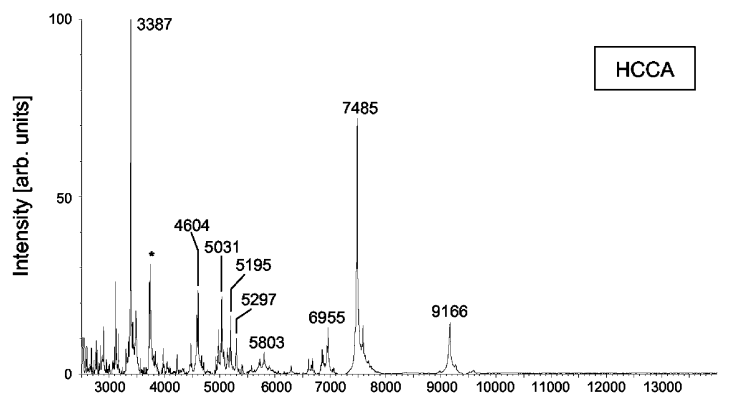

(d)
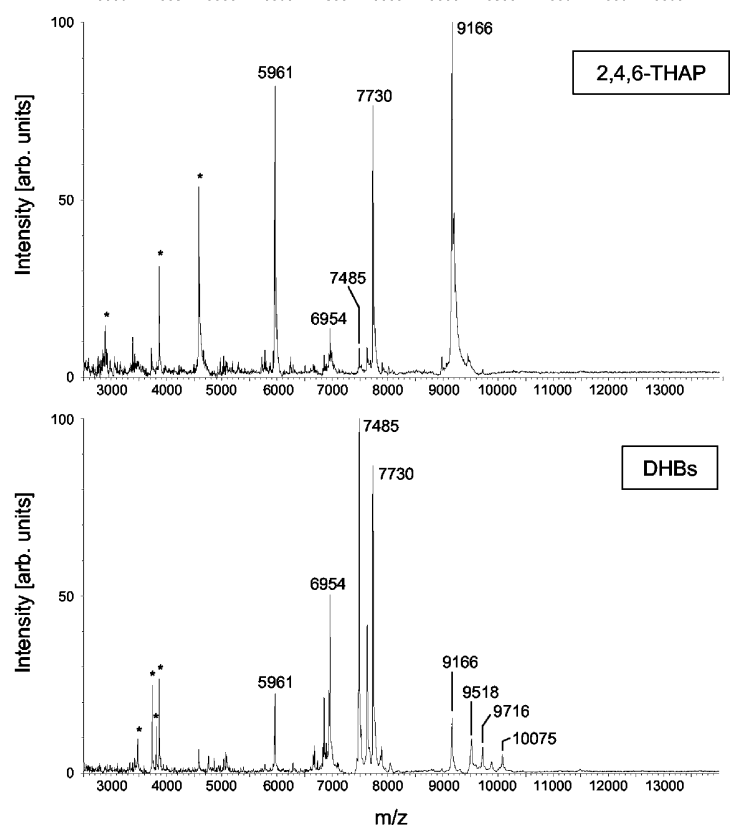

Figure 5. Influence of the matrix on MALDI mass spectra of wet heat-treated $\left(20 \mathrm{~min}\right.$ at $\left.120^{\circ} \mathrm{C}\right) \mathrm{B}$. subtilis strain RL45. The following matrices were used: (a) SA, (b) HCCA, (c) 2,4,6-THAP, and (d) DHBs. The two-layer method was used as preparation technique. The asterisk indicates peak of doubly charged species.

specific for neither matrix nor fragment masses and (2) equivalent adducts of the other peaks in lower $\mathrm{m} / \mathrm{z}$ range are missing. The major high-mass biomarker masses are added to Table 1 for comparison of the different strains analyzed.

Unfortunately, only little is known about matrix effects in the analysis of intact bacterial spores. Elhanany et al. who compared SA and HCCA observed a similar behavior during the analysis of spore extracts: HCCA generally tends to give mass spectra in which biomarkers with lower masses are more pronounced while SA appears to be more suitable for high-mass biomarkers [12]. Our observations are in good agreement to this. Ramirez and Fenselau investigated the influence of SA, HCCA, and 2,5-DHB on the peak broadening of biomarkers desorbed from spores of $B$. thuringiensis [46]. As they used a low mass lipopeptide (Kurstakin, 906.2u) as biomarker molecule, no comparable data for the high-mass biomarkers are available. For the analysis of fungal spores, inconsistent results are described for the influence of matrices on the mass spectrum. Armiri et al. compared SA, HCCA, ferulic acid, 3-hydroxypicolinic acid, and 2,5-DHB with the conclusion that SA performs best and 2,5-DHB worst [47], whereas Valentine et al. found ferulic acid to be a better matrix than SA [48]. Li et al. who studied amongst others the feasibility of SA and HCCA, described that both matrices are equally suitable for the analysis of fungal spores [49]. Welham et al. chose SA and 2,5-DHB as best matrices, especially for detecting high-mass biomarkers. They describe 2,6-dihydroxyacetophenon, which is an alternative for 2,4,6-THAP, as an unsuitable matrix [50]. Similar inconsistent observations are described for the analysis of whole bacterial cells $[8,27,28,51-54]$. Unfortunately, these results are difficult to compare due to strong structural differences between bacterial spores and their vegetative cells or fungal spores. However, not only different matrices lead to different results. It was observed reproducibly that different batches, i.e., different suppliers and lots, of the same matrix substance lead to different peak pattern-for example dramatically different $\mathrm{S} / \mathrm{N}$ ratio (spectra not shown). Nevertheless, these controversial findings show that the selection of matrix for the MALDI-MS analysis of complex biomaterials, is difficult. Even in (more fundamental) studies of much simpler systems-like the analysis of just one protein-it is still unclear which experimental conditions are necessary for a successful and reproducible MALDI process $[30,31,55,56]$. Until this process is understood in more detail, a careful standardization and detailed description of matrix choice and sample preparation technique is required.

\section{Sample Concentration}

In a recent study, a dramatic influence of slight variations in sample concentration on the peak appearance in the MALDI spectra of intact bacterial cells was described [8]. As it is unknown how much biomarker material is accessible from the intact spores after the wet heat-treatment and whether a comparable amount is released for every strain, it was investigated how the peak patterns change with spore concentration. Series of ten-time dilutions were prepared for selected samples of different wet heat-treated strains starting with a concentration of approximately $10^{9}$ spores $/ \mathrm{ml}$. As an example a typical high-mass biomarker peak pattern is given in Figure 6. The peak pattern appeared to be stable over a concentration range of at least one order of 
(a)

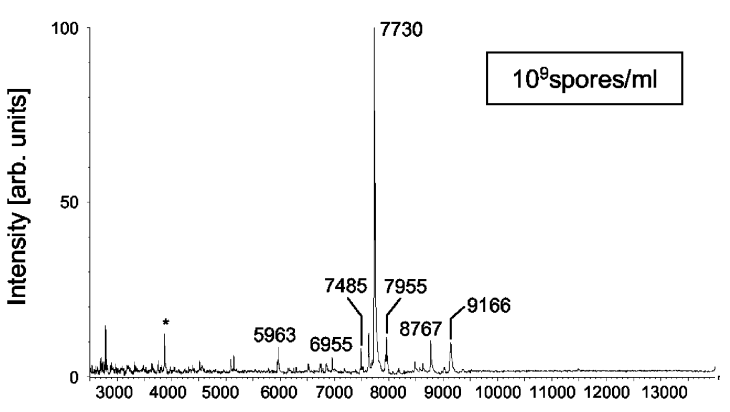

(b)

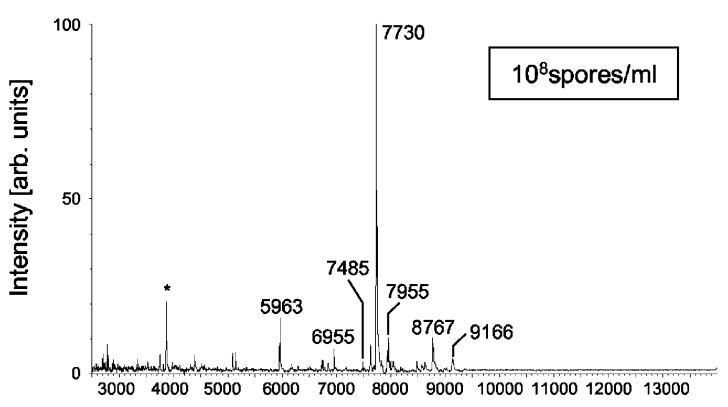

(c)

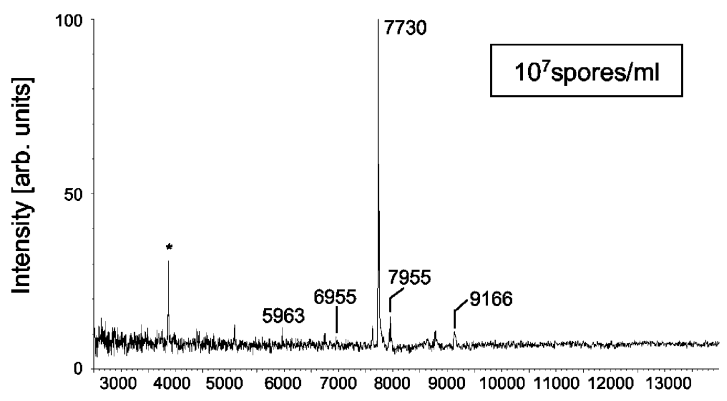

(d)

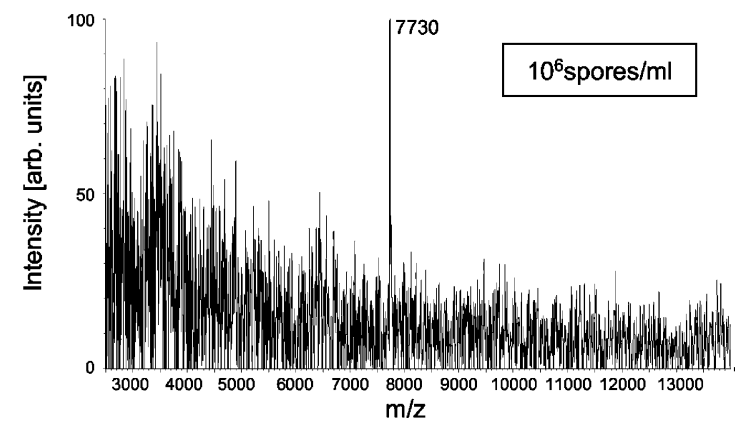

Figure 6. Influence of the concentration on MALDI mass spectra of untreated and wet heat-treated $\left(3 \mathrm{~min}\right.$ at $\left.120^{\circ} \mathrm{C}\right) \mathrm{B}$. subtilis strain RL45. 10-fold dilutions were used as follows: (a) original concentration of about $10^{9}$ spores $/ \mathrm{ml}$, (b) 1:10 dilution, (c) 1:100, and (d) 1:1000. SA was used as matrix and the inverse two-layer method as preparation technique. The asterisk indicates peaks of doubly charged species.

magnitude up to a concentration of $10^{8}$ spores $/ \mathrm{ml}$. This concentration is in the same range as used by Hathout et al. $\left(2.4 \times 10^{8}\right.$ spores $\left./ \mathrm{ml}\right)$ [13]. A decrease in concentration led to a proportional decrease in the $\mathrm{S} / \mathrm{N}$ ratio. This is in good agreement with the general behavior in MALDI-MS, e.g., for pure protein solutions. Based on these observations, the suitability of MALDI-MS for quantitative analysis of the peak pattern in the analysis of intact bacterial spores can be speculated. A more comprehensive study concerning this subject is in preparation [22].

\section{Influence of Microbiological Parameters}

The influence of different microbiological handling of spores, e.g., sporulation medium, washing procedure, and storage, was examined as these parameters might influence the high-mass biomarker peak pattern.

\section{Sporulation Media}

In order to get a first impression whether the sporulation medium has an influence on the peak pattern, two Bacilli strains (MC85 and PEA26) were grown and sporulated on three different media (nutrient agar, Schaeffer's, BHI). The harvested spores were wet heattreated by boiling aliquots for $20 \mathrm{~min}$ at $100{ }^{\circ} \mathrm{C}$ to release the biomarkers. They were analyzed by means of SA and the inverse two-layer method. The results differ for the two Bacillus species tested. While the growth medium did not seem to have an influence on the peak pattern of PEA26 (data not shown), the peak pattern of MC85 changes with the used sporulation medium as seen in Figure 7. In general, the spectra differ dramatically in peak appearance as well as in relative peak intensity. This behavior is in good agreement with observations made by Ryzhov et al. who compared the peak pattern of B. thuringiensis subsp. kurstaki and B. cereus sporulated on three different media for high-mass biomarker [14]. This effect makes it more difficult to compare the high-mass biomarker peak pattern of spores, even if species and strain are similar or even identical. A further standardization of the growth conditions might be helpful in inter-laboratory comparisons. Nevertheless, a more thorough study is needed to evaluate this influence for the feasibility of spectral analysis by library searching. Here, the addition of reference spectra for each medium can be a first approach as suggested by Ryzhov et al. [14]. However, this is limited to standardized microbiological growth media and, for instance, will fall short for spores formed in industrial food production.

\section{Washing Procedure After Wet Heat-Treatment}

A probable future application of the MALDI-MS analysis would be the detection of intact bacterial spores in food products. Here, components of the food matrix might be absorbed on the spore surface and interfere with the analysis of the high-mass biomarker peak pattern. Therefore, a cleaning procedure has to be performed. The high-mass biomarker peak patterns of unwashed wet heat-treated spores were compared to those obtained after washing the wet heat-treated spores up to three times with deionized water. In general, the same peaks can be found for both, washed and unwashed, samples but the relative peak intensities of high-mass biomarkers can be influenced by the 
(a)

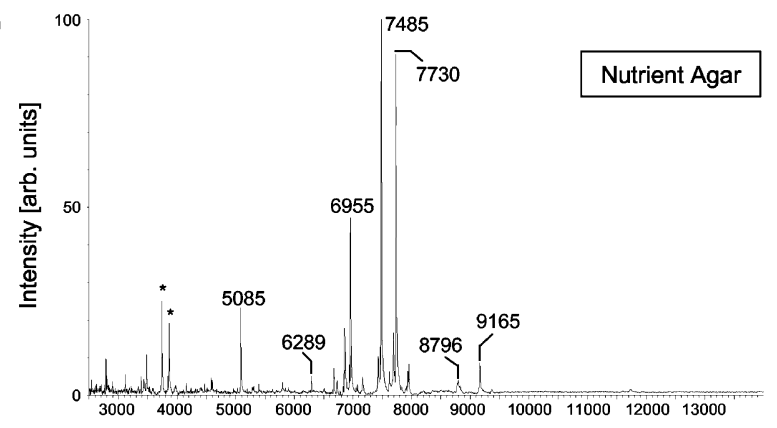

(b)

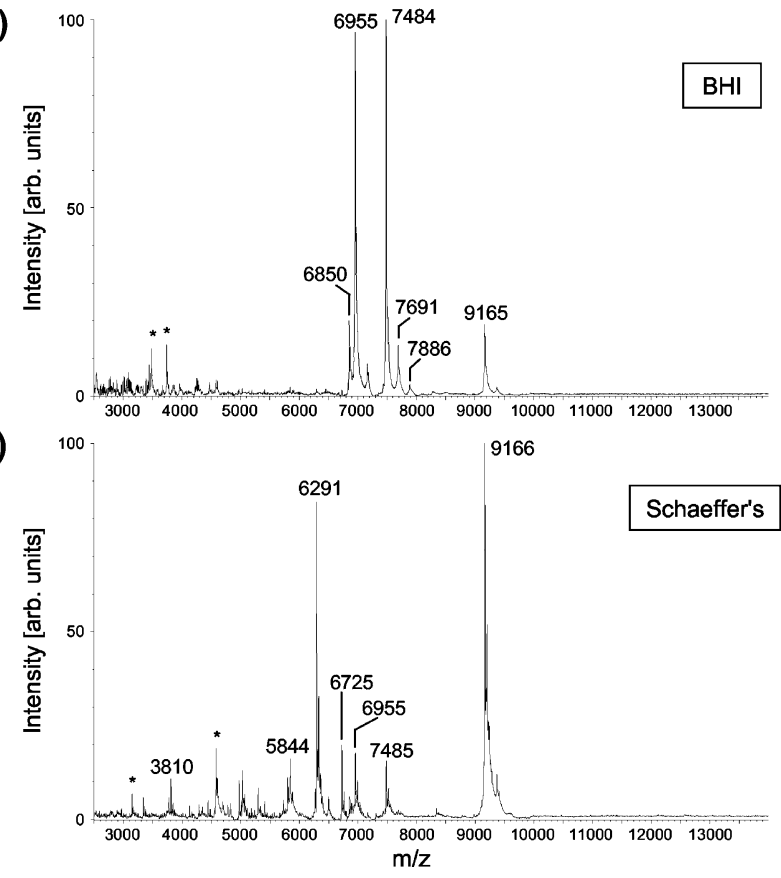

Figure 7. Influence of the growth medium on MALDI mass spectra of wet heat-treated $\left(20 \mathrm{~min}\right.$ at $\left.120^{\circ} \mathrm{C}\right)$ B. subtilis strain MC85. Growth media are, (a) nutrient agar, (b) BHI, and (c) Schaeffer's sporulation medium. SA was used as matrix and the inverse two-layer method as preparation technique. The asterisk indicates peak of doubly charged species.

washing procedure quite dramatic. This effect appeared to be strain-specific. While the high-mass peak pattern was stable for spores of $B$. cereus strain PEA26 the high-mass biomarkers appearing at $\mathrm{m} / \mathrm{z} 6955$ and 7485 of B. subtilis strain MC85 could be washed off easily (data not shown). Similar to Hathout et al. who concluded that the washing-off by organic solvents is related to the lipophilic character of the other high-mass biomarker molecules [13], we explain the observed behavior by the hydrophilic character of the SASPs.

\section{Storage Conditions}

Aliquots of untreated and wet heat-treated spore solutions were stored at $4{ }^{\circ} \mathrm{C}$ and $-80^{\circ} \mathrm{C}$ for at least a month. Then, the frozen spore solutions were thawed and analyzed by MALDI-MS. No differences in peak pattern were observed for the different storage conditions (data not shown).

\section{Conclusions}

This study presented short wet heat-treatment as a new technique to release high-mass biomarkers to obtain fingerprints of intact bacterial spores by MALDI-MS. This new releasing technique is straightforward and easily applicable in every laboratory. It appears to be less destructive than other techniques applied so far, which allows further investigation of the spores. The influence of specific experimental changes, e.g., choice of matrix and sample preparation, and microbiological handling, e.g., use of sporulation media, on the final high-mass biomarker peak pattern after wet heat-treatment as releasing technique was shown to be substantial. Standardized protocols can be a first opportunity to enable inter-laboratory comparison and automatic industrial application. Our results revealed that a wet heat-treatment of bacterial spores at $100{ }^{\circ} \mathrm{C}$ for 10 to 20 min and subsequent analysis using the inverse twolayer method and SA lead to mass spectra of appropriate quality reproducibly. Irrespective which releasing technique is used, the observed high-mass biomarkers seem to belong to the group of small, acid-soluble proteins, the SASPs, which seem to be very good markers for routine taxonomy of bacterial spores.

\section{Acknowledgments}

Pieter ter Steeg, Unilever Vlaardingen, is gratefully acknowledged for his idea of analyzing heat-treated spores with MALDI-MS. VH was supported by a Marie Curie Fellowship of the European Community program Quality of Life under contract number QLK3-CT-2001-60028. RN was supported by the Federal Office for Education and Research (Bundesamt für Bildung und Wissenschaft) of Switzerland under the BBW-reference number 02.040. Wilma du Chatinier is acknowledged for her help with the TOFSpec 2E.

\section{References}

1. Anhalt, J. P.; Fenselau, C. Identification of Bacteria Using Mass Spectrometry. Anal. Chem. 47, 1975, 219-225.

2. Beverly, M. B.; Basile, F.; Voorhees, K. J.; Hadfield, T. L. A Rapid Approach for the Detection of Dipicolinic Acid in Bacterial Spores Using Pyrolysis Mass Spectrometry. Rapid Commun. Mass Spectrom. 1996, 10, 455-458.

3. Cain, T. C.; Lubman, D. M.; Weber, W. J. Differentiation of Bacteria Using Protein Profiles from Matrix-Assisted Laser Desorption/Ionization Time-of-Flight Mass Spectrometry. Rapid Commun. Mass Spectrom. 1994, 8, 1026-1030.

4. Claydon, M. A.; Davey, S. N.; Edwards-Jones, V.; Gordon, D. B. The Rapid Identification of Intact Microorganisms Using Mass Spectrometry. Nat. Biotechnol. 1996, 14, 1584-1586.

5. Holland, R. D.; Wilkes, J. G.; Rafii, F.; Sutherland, J. B.; Persons, C. C.; Voorhees, K. J.; Lay, J. O. Rapid Identification of Intact Whole Bacteria Based on Spectral Patterns Using Matrix-Assisted Laser Desorption/Ionization with Time-ofFlight Mass Spectrometry. Rapid Commun. Mass Spectrom. 1996, 10, 1227-1232.

6. Krishnamurthy, T.; Ross, P. L.; Rajamani, U. Detection of Pathogenic and Non-Pathogenic Bacteria by Matrix-Assisted Laser Desorption/Ionization Time-of-Flight Mass Spectrometry. Rapid Commun. Mass Spectrom. 1996, 10, 883-888. 
7. Krishnamurthy, T.; Ross, P. L. Rapid Identification of Bacteria by Direct Matrix-Assisted Laser Desorption/Ionization Mass Spectrometric Analysis of Whole Cells. Rapid Commun. Mass Spectrom. 1996, 10, 1992-1996.

8. Williams, T. L.; Andrzejewski, D.; Lay, J. O.; Musser, S. M. Experimental Factors Affecting the Quality and Reproducibility of MALDI TOF Mass Spectra Obtained from Whole Bacteria Cells. J. Am. Soc. Mass Spectrom. 2003, 14, 342-351.

9. Setlow, P.; Johnson, E. A. Spores and Their Significance. In Food Microbiology Fundamentals and Frontier; Doyle, M. P.; Beuchart, L. R.; Montville, T. J.; ASM Press: Washington DC., 1996, pp 30-65.

10. Beverly, M. B.; Voorhees, K. J.; Hadfield, T. L. Direct Mass Spectrometric Analysis of Bacillus Spores. Rapid Commun. Mass Spectrom. 1999, 13, 2320-2326.

11. Birmingham, J.; Demirev, P.; Ho, Y. P.; Thomas, J.; Bryden, W.; Fenselau, C. Corona Plasma Discharge for Rapid Analysis of Microorganisms by Mass Spectrometry. Rapid Commun. Mass Spectrom. 1999, 13, 604-606.

12. Elhanany, E.; Barak, R.; Fisher, M.; Kobiler, D.; Altboum, Z. Detection of Specific Bacillus Anthracis Spore Biomarkers by Matrix-Assisted Laser Desorption/Ionization Time-of-Flight Mass Spectrometry. Rapid Commun. Mass Spectrom. 2001, 15, 2110-2116.

13. Hathout, Y.; Demirev, P. A.; Ho, Y. P.; Bundy, J. L.; Ryzhov, V.; Sapp, L.; Stutler, J.; Jackman, J.; Fenselau, C. Identification of Bacillus Spores by Matrix-Assisted Laser Desorption/Ionization Mass Spectrometry. Appl. Environ. Microbiol. 1999, 65, 4313-4319.

14. Ryzhov, V.; Hathout, Y.; Fenselau, C. Rapid Characterization of Spores of Bacillus Cereus Group Bacteria by Matrix-Assisted Laser Desorption/Ionization Time-of-Flight Mass Spectrometry. Appl. Environ. Microbiol. 2000, 66, 3828-3834.

15. Hathout, Y.; Setlow, B.; Cabrera-Martinez, R. M.; Fenselau, C.; Setlow, P. Small, Acid-Soluble Proteins as Biomarkers in Mass Spectrometry Analysis of Bacillus Spores. Appl. Environ. Microbiol. 2003, 69, 1100-1107.

16. Afonso, C.; Fenselau, C. Use of Bioactive Glass Slides for Matrix-Assisted Laser Desorption/Ionization Analysis: Application to Microorganisms. Anal. Chem. 2003, 75, 694-697.

17. Cazemier, A. E.; Wagenaars, S. F. M.; ter Steeg, P. F. Effect of Sporulation and Recovery edium on the Heat Resistance and Amount of Injury of Spores from Spoilage Bacilli. J. Appl. Microbiol. 2001, 90, 761-770.

18. Schaeffer, P. J.; Millet, J.; Aubert, J.-P. Catabolic Repression of Bacterial Sporulation. Proc. Natl. Acad. Sci. U.S.A. 1965, 54, 704-711.

19. Kooiman, W. J. The Screw Cap Tube Technique: A New and Accurate Technique for the Determination of the Wet Heat Resistance of Bacterial Spores. In Spore Research; Barker, A. N.; Gould, G. W.; Wolf, J.; Academic Press: London, 1973, pp 87-92.

20. Vorm, O.; Roepstorff, P.; Mann, M. Improved Resolution and Very High-Sensitivity in MALDI TOF of Matrix Surfaces Made by Fast Evaporation. Anal. Chem. 1994, 66, 3281-3287.

21. Strupat, K.; Karas, M.; Hillenkamp, F. 2,5-Dihydroxybenzoic Acid-A New Matrix for Laser Desorption/Ionization Mass Spectrometry. Int. J. Mass Spectrom. Ion Processes 1991, 111, 89-102.

22. Notz, R. E.; Haverkamp, J.; Horneffer, V., unpublished.

23. Demirev, P. A.; Ramirez, J.; Fenselau, C. Tandem Mass Spectrometry of Intact Proteins for Characterization of Biomarkers from Bacillus cereus T Spores. Anal. Chem. 2001, 73, 5725-5731.

24. Leuschner, R. G. K.; Lillford, P. J. Investigation of Bacterial Spore Structure by High Resolution Solid-State Nuclear Magnetic Resonance Spectroscopy and Transmission Electron Microscopy. Int. J. Food Microbiol. 2001, 63, 35-50.
25. Gould, G. W. Bacterial Endospores. In Encycopedia of Food Microbiology; Robinson, R. K.; Batt, C. A.; Patel, P. D., Eds.; Academic Press: London, 2000; pp 168-172.

26. Saenz, A. J.; Petersen, C. E.; Valentine, N. B.; Gantt, S. L.; Jarman, K. H.; Kingsley, M. T.; Wahl, K. L. Reproducibility of Matrix-Assisted Laser Desorption/Ionization Time-of-Flight Mass Spectrometry for Replicate Bacterial Culture Analysis. Rapid Commun. Mass Spectrom. 1999, 13, 1580-1585.

27. Evason, D. J.; Claydon, M. A.; Gordon, D. B. Effects of Ion Mode and Matrix Additives in the Identification of Bacteria by Intact Cell Mass Spectrometry. Rapid Commun. Mass Spectrom. 2000, 14, 669-672.

28. Madonna, A. J.; Basile, F.; Ferrer, I.; Meetani, M. A.; Rees, J. C.; Voorhees, K. J. On-Probe Sample Pretreatment for the Detection of Proteins Above $15 \mathrm{KDa}$ from Whole Cell Bacteria by Matrix-Assisted Laser Desorption/Ionization Time-of-Flight Mass Spectrometry. Rapid Commun. Mass Spectrom. 2000, 14, 2220-2229.

29. Vaidyanathan, S.; Winder, C. L.; Wade, S. C.; Kell, D. B.; Goodacre, R. Sample Preparation in Matrix-Assisted Laser Desorption/Ionization Mass Spectrometry of Whole Bacterial Cells and the Detection of High Mass ( $>20 \mathrm{KDa}$ ) Proteins. Rapid Commun. Mass Spectrom. 2002, 16, 1276-1286.

30. Beavis, R. C.; Bridson, J. N. Epitaxial Protein Inclusion in Sinapic Acid Crystals. J. Phys. D Appl. Phys. 1993, 26, 442-447.

31. Horneffer, V.; Dreisewerd, K.; Lüdemann, H. C.; Hillenkamp, F.; Läge, M.; Strupat, K. Is the Incorporation of Analytes into Matrix Crystals a Prerequisite for Matrix-Assisted Laser Desorption/Ionization Mass Spectrometry? A Study of Five Positional Isomers of Dihydroxybenzoic Acid. Int. J. Mass Spectrom. 1999, 187, 859-870.

32. Horneffer, V.; Forsmann, A.; Strupat, K.; Hillenkamp, F.; Kubitscheck, U. Localization of Analyte Molecules in MALDI Preparations by Confocal Laser Scanning Microscopy. Anal. Chem. 2001, 73, 1016-1022.

33. Glückmann, M.; Pfenninger, A.; Krüger, R.; Thierolf, M.; Karas, M.; Horneffer, V.; Hillenkamp, F.; Strupat, K. Mechanisms in MALDI Analysis: Surface Interaction or Incorporation of Analytes? Int. J. Mass Spectrom. 2001, 210, 121-132.

34. Andersson, A.; Ronner, U. Adhesion and Removal of Dormant, Heat-Activated, and Germinated Spores of Three Strains of Bacillus cereus. Biofouling 1998, 13, 51-67.

35. Peng, J. S.; Tsai, W. C.; Chou, C. C. Surface Characteristics of Bacillus cereus and Its Adhesion to Stainless Steel. Int. J. Food Microbiol. 2001, 65, 105-111.

36. Ryzhov, V.; Bundy, J. L.; Fenselau, C.; Taranenko, N.; Doroshenko, V.; Prasad, C. R. Matrix-Assisted Laser Desorption/ Ionization Time-of-Flight Analysis of Bacillus Spores Using a $2.94 \mu \mathrm{m}$ Infrared Laser. Rapid Commun. Mass Spectrom. 2000, 14, 1701-1706.

37. Beavis, R. C.; Chaudhary, T.; Chait, B. T. $\alpha$-Cyano-4-hydroxycinnamic Acid as a Matrix for Matrix-Assisted Laser Desorption Mass Spectrometry. Org. Mass Spectrom. 1992, 27, 156-158.

38. Rosinke, B.; Strupat, K.; Hillenkamp, F.; Rosenbusch, J.; Dencher, N.; Krüger, U.; Galla, H. J. Matrix-Assisted Laser Desorption/Ionization Mass Spectrometry (MALDI-MS) of Membrane-Proteins and Noncovalent Complexes. J. Mass Spectrom. 1995, 30, 1462-1468.

39. Zhu, Y. F.; Chung, C. N.; Taranenko, N. I.; Allman, S. L.; Martin, S. A.; Haff, L.; Chen, C. H. The Study of 2,3,4Trihydroxyacetophenone and 2,4,6- Trihydroxyacetophenone as Matrices for DNA Detection in Matrix-Assisted Laser Desorption/Ionization Time-of-Flight Mass Spectrometry. Rapid Commun. Mass Spectrom. 1996, 10, 383-388.

40. Stahl, B.; Steup, M.; Karas, M.; Hillenkamp, F. Analysis of Neutral Oligosaccharides by Matrix-Assisted Laser Desorp- 
tion/Ionization Mass Spectrometry. Anal. Chem. 1991, 63, 1463-1466.

41. Karas, M.; Ehring, H.; Nordhoff, E.; Stahl, B.; Strupat, K.; Hillenkamp, F.; Grehl, M.; Krebs, B. Matrix-Assisted Laser Desorption/Ionization Mass Spectrometry with Additives to 2,5-Dihydroxybenzoic Acid. Org. Mass Spectrom. 1993, 28, 1476-1481.

42. Strupat, K.; Kampmeier, J.; Horneffer, V. Investigations of 2,5-DHB and Succinic Acid as Matrices for UV and IR MALDI. Part II. Crystallographic and Mass Spectrometric Analysis. Int. J. Mass Spectrom. 1997, 169, 43-50.

43. Horneffer, V.; Reichelt, R.; Strupat, K. Protein Incorporation into MALDI Matrix Crystals Investigated by High Resolution Field Emission Scanning Electron Microscopy. Int. J. Mass Spectrom. 2003, 226, 117-131.

44. Dai, Y. Q.; Whittal, R. M.; Li, L. Confocal Fluorescence Microscopic Imaging for Investigating the Analyte Distribution in MALDI Matrices. Anal. Chem. 1996, 68, 2494-2500.

45. Fournier, I.; Tabet, J. C.; Bolbach, G. Irradiation Effects in MALDI and Surface Modifications. Part I. Sinapinic Acid Monocrystals. Int. J. Mass Spectrom. 2002, 219, 515-523.

46. Ramirez, J.; Fenselau, C. Factors Contributing to Peak Broadening and Mass Accuracy in the Characterization of Intact Spores Using Matrix-Assisted Laser Desorption/Ionization Coupled with Time-of-Flight Mass Spectrometry. J. Mass Spectrom. 2001, 36, 929-936.

47. Amiri-Eliasi, B. J.; Fenselau, C. Characterization of Protein Biomarkers Desorbed by MALDI from Whole Fungal Cells. Anal. Chem. 2001, 73, 5228-5231.

48. Valentine, N. B.; Wahl, J. H.; Kingsley, M. T.; Wahl, K. L. Direct Surface Analysis of Fungal Species by Matrix-Assisted Laser
Desorption/Ionization Mass Spectrometry. Rapid Commun. Mass Spectrom. 2002, 16, 1352-1357.

49. Li, T. Y.; Liu, B. H.; Chen, Y. C. Characterization of Aspergillus Spores by Matrix-Assisted Laser Desorption/Ionization Timeof-Flight Mass Spectrometry. Rapid Commun. Mass Spectrom. 2000, 14, 2393-2400.

50. Welham, K. J.; Domin, M. A.; Johnson, K.; Jones, L.; Ashton, D. S. Characterization of Fungal Spores by Laser Desorption/ Ionization Time-of-Flight Mass Spectrometry. Rapid Commun. Mass Spectrom. 2000, 14, 307-310.

51. Easterling, M. L.; Colangelo, C. M.; Scott, R. A.; Amster, I. J. Monitoring Protein Expression in Whole Bacterial Cells with MALDI Time-of-Flight Mass Spectrometry. Anal. Chem. 1998, 70, 2704-2709.

52. Domin, M. A.; Welham, K. J.; Ashton, D. S. The Effect of Solvent and Matrix Combinations on the Analysis of Bacteria by Matrix-Assisted Laser Desorption/Ionization Time-ofFlight Mass Spectrometry. Rapid Commun. Mass Spectrom. 1999, 13, 222-226.

53. Ryzhov, V.; Fenselau, C. Characterization of the Protein Subset Desorbed by MALDI from Whole Bacterial Cells. Anal. Chem. 2001, 73, 746-750.

54. Bright, J. J.; Claydon, M. A.; Soufian, M.; Gordon, D. B. Rapid Typing of Bacteria Using Matrix-Assisted Laser Desorption/ Ionization Time-of-Flight Mass Spectrometry and Pattern Recognition Software. J. Microbiol. Methods 2002, 48, 127-138.

55. Karas, M.; Glückmann, M.; Schäfer, J. Ionization in MatrixAssisted Laser Desorption/Ionization: Singly Charged Molecular Ions are the Lucky Survivors. J. Mass Spectrom. 2000, 35, $1-12$.

56. Knochenmuss, R.; Zenobi, R. MALDI Ionization: The Role of in-Plume Processes. Chem. Rev. 2003, 103, 441-452. 